Compartmental Modeling Of The Fluorescence Anisotropy Decay Of A Cylindrically Symmetric Brownian Rotor: Identifiability Analysis.

Non Peer-reviewed author version

Boens, N.; Novikov, E. \& AMELOOT, Marcel (2006) Compartmental Modeling Of The Fluorescence Anisotropy Decay Of A Cylindrically Symmetric Brownian Rotor: Identifiability Analysis.. In: CHEMPHYSCHEM, 7. p. 2559-2566.

DOI: $10.1002 /$ cphc.200600309

Handle: http://hdl.handle.net/1942/1679 


\section{Compartmental Modeling of the Fluorescence Anisotropy Decay of a Cylindrically Symmetric Brownian Rotor. Identifiability Analysis}

Revised: July 21, 2006

Noël Boens, ${ }^{a,{ }^{*}}$ Eugene Novikov, ${ }^{b}$ Marcel Ameloot ${ }^{c}$

${ }^{a}$ Department of Chemistry, Katholieke Universiteit Leuven, Celestijnenlaan $200 \mathrm{~F}$, 3001 Heverlee, Belgium

${ }^{b}$ Institut Curie, Service Bioinformatique, 26 Rue d'Ulm, Paris Cedex 05, 75248 France

${ }^{c}$ Hasselt University, Biomedical Research Institute, Agoralaan, Building D, 3590 Diepenbeek, Belgium

*Corresponding author: Noel.Boens@chem.kuleuven.be

We present the results of the deterministic identifiability analysis based on similarity transformation for models of one-state excited-state events of cylindrically symmetric rotors in isotropic environments undergoing rotational diffusion described by Brownian reorientation. Such an analysis on error-free time-resolved fluorescence (anisotropy) data can reveal whether the parameters of the considered model can be determined. The fluorescence $\delta$-response functions $I_{\|}(t)$ and $I_{\perp}(t)$, for fluorescence polarized respectively parallel and perpendicular to the electric vector of linearly polarized excitation, are used to construct, in convenient matrix form, expressions of the sum $S(t)=I_{\|}(t)+2 I_{\perp}(t)$, the difference $D(t)=I_{\|}(t)-I_{\perp}(t)$, and the time-resolved fluorescence anisotropy $r(t)=\mathrm{D}(t) / S(t)$. The identifiability analysis of $r(t)$ demonstrates that the rotational diffusion coefficients $D_{\|}$and $D_{\perp}$ for rotation respectively about and perpendicular to the symmetry axis can be uniquely 
determined. However, the polar and azimuthal angles defining the absorption and emission transition moments in the molecular reference frame are not uniquely determined. Nevertheless, the difference between the polar angles of these transition moments is uniquely determined.

\section{Introduction}

Time-resolved fluorescence depolarization is a very powerful tool for obtaining information about not only the overall excited-state dynamics of a fluorophore but also its rotational motions. Information about the shape of a molecule, as well as its interaction with the surrounding environment, can be obtained. For nonspherical molecules, the fluorescence anisotropy decay is generally highly complex, even in the absence of extra complicating factors such as excited-state reactions, energy transfer, solvent interactions, etc. In this paper we will consider the time-dependence of the fluorescence anisotropy of a fluorophore modeled as a cylindrically symmetric rotor undergoing rotational diffusion in an isotropic environment. The emphasis is on a compartmental approach leading to a matrix description of the time-resolved anisotropy and on the identifiability analysis of the considered models.

Whenever a specific model is proposed for the description of excited-state processes, one should start with a study to verify whether the fundamental kinetic, spectral, shape, and orientation parameters defining the model can be determined from errorfree (i.e., perfect) fluorescence (anisotropy) decay data. This is the object of deterministic identifiability analysis. ${ }^{[1],[2],[3],[4]}$ For models of one-state excited-state processes coupled with rotational diffusion - considered in this paper - the parameters to be identified are the deactivation rate constant, the rotational diffusion coefficients, and the orientations of the absorption and emission transition moments within the molecular reference frame. 
A deterministic identifiability analysis is a prerequisite before attempting to estimate numerical values of the model parameters from real experimental observations. Large uncertainties and high correlations obtained in the parameter recovery from an experimental data surface might erroneously be ascribed to numerical ill-conditioning, because they may rather be indicative of the fact that the model parameters cannot be recovered at all (i.e., the model is not identifiable). Imperfect data resulting from noisy observations sampled over a limited time range affect the accuracy and precision with which model parameters can be estimated. This numerical parameter estimation and the statistical properties of the parameter estimates are the subject of the second stage of any identifiability analysis and are called numerical identifiability. A study of the data analysis (i.e., curve-fitting), which takes into account the noise level on the experimental data, the sampling, and the sensitivity of the algorithms used in the estimation of the parameters, is outside the scope of this paper, however.

Since the first identifiability analysis of a model for excited-state processes, ${ }^{[5],[6],[7]}$ identifiability studies of a large range of photophysical models have been reported (see references in [4] and [8] for literature data). In the extensive field of timeresolved fluorescence spectroscopy, only a rather small part of the literature has been devoted to excited-state processes coupled with species-dependent rotational diffusion (see [9] and references therein). Up to now, three identifiability analyses of models for excited-state events coupled with rotational diffusion have been reported. ${ }^{[9],[10],[11]}$ This report focuses on the identifiability of models for one-state excited-state processes, accompanied by rotational diffusion described by Brownian reorientation, as detected by time-resolved fluorescence anisotropy. Cylindrically and spherically symmetric rotors are considered. Derivations of the time-resolved fluorescence anisotropy of a totally asymmetric rotor using the diffusion equation ${ }^{[12],[13],[14]}$ are 
based on the rotational diffusion equation given by Favro. ${ }^{[15]}$ The basis for the derivation of the explicit expressions describing the time-resolved fluorescence anisotropy of cylindrically symmetric rotors has been provided by Chuang and Eisenthal $^{[12]}$, by Ehrenberg and Rigler ${ }^{[13]}$, and by Belford et al. ${ }^{[14]} \operatorname{Razi~Naqvi}^{[16]}$ demonstrated that the time-resolved fluorescence depolarization can be reformulated in terms of conventional reaction kinetics by showing the equivalence to a set of ordinary linear differential equations. Piston and Gratton ${ }^{17}$ have extended, in a compartmental formalism, the jump model between predefined orthogonal orientations for the general, asymmetric rotor, originally developed by Weber. ${ }^{18}$ The compartmental analysis approach has the merit that additional processes contributing to the anisotropy decay can be readily incorporated. Starting from the compartmental models reported in references [9]-[11], which are based on the rotational diffusion equation, we describe explicitly - in matrix form - the fluorescence anisotropy decay of one-state excited-state events accompanied by rotational diffusion of cylindrically symmetric species.

The paper is organized as follows. Section 2 gives an outline - in terms of a compartmental model - of the derivation of the expression for the fluorescence anisotropy decay $r(t)$ of one-state excited-state processes coupled with rotational diffusion described by Brownian reorientation for cylindrically and spherically symmetric ellipsoids. The anisotropy $r(t)$ is expressed in matrix form, suitable for the identifiability analysis based on similarity transformations. Section 3 deals with the deterministic identifiability analysis of the kinetic models considered. The similarity transformation used for this purpose can be applied for reactions in general. 


\section{Kinetics}

Here we give an outline of the derivation - based on the compartmental approach described in reference [9] - of the kinetic expression of the fluorescence anisotropy in matrix form, needed for the identifiability analysis.

Consider the linear, time-invariant photophysical system consisting of ground-state species 1 with distinct rotational characteristics, as depicted in Figure 1. Photoexcitation produces the excited-state species $\mathbf{1}^{*}$ which can decay by fluorescence $\left(k_{\mathrm{F}}\right)$ and nonradiative $\left(k_{\mathrm{NR}}\right)$ processes, characterized by the composite deactivation rate constant $k_{01}\left(=k_{\mathrm{F}}+k_{\mathrm{NR}}\right)$. Physical requirements confine the rate constants $k_{\mathrm{F}}$ and $k_{\mathrm{NR}}$, and therefore also $k_{01}$, to be nonnegative. In the particular case of prolate and oblate ellipsoids, the rotational relaxation of the excited-state species $\mathbf{1}^{*}$ is described by the rotational diffusion coefficients $D_{\|}$and $D_{\perp}$, also physically limited to positive values, for rotations about the symmetry axis and the equatorial axes, respectively (Figure 1).

\section{$<$ Figure $1>$}

When the photophysical system shown in Figure 1 is photo-excited at time zero with a $\delta$-pulse of wavelength $\lambda_{i}^{\text {ex }}$ of low intensity, so that the ground-state species population is not appreciably depleted, the fluorescence $\delta$-response function $I_{\| i j}(t)$ observed at wavelength $\lambda_{j}^{\mathrm{ex}}$ for the plane-polarized component of emission of $\mathbf{1}^{*}$, having its electric vector polarized parallel to the electric vector of the plane-polarized excitation light, and the fluorescence $\delta$-response function $I_{\perp i j}(t)$ for the perpendicularly polarized component can be expressed, in the case of pure transitions and isotropic environments, as ${ }^{[19],[20]}$

$$
I_{\| i j}(t)=\frac{1}{3} S_{i j}(t)\left[1+2 r_{i j}(t)\right]=\frac{1}{3}\left[S_{i j}(t)+2 D_{i j}(t)\right], \quad t \geq 0
$$




$$
I_{\perp i j}(t)=\frac{1}{3} S_{i j}(t)\left[1-r_{i j}(t)\right]=\frac{1}{3}\left[S_{i j}(t)-D_{i j}(t)\right], \quad t \geq 0
$$

where $r_{i j}(t)$ denotes the time-dependent fluorescence anisotropy:

$$
r_{i j}(t)=\frac{D_{i j}(t)}{S_{i j}(t)}, \quad t \geq 0
$$

and where $S_{i j}(t)$ and $D_{i j}(t)$ are defined by ${ }^{[19],[20]}$

$$
\begin{aligned}
& S_{i j}(t)=I_{\|_{i j}}(t)+2 I_{\perp i j}(t), \quad t \geq 0 \\
& D_{i j}(t)=I_{\|_{i j}}(t)-I_{\perp i j}(t), \quad t \geq 0
\end{aligned}
$$

The subscripts $i$ and $j$ in $I_{\| i j}(t), I_{\perp i j}(t), S_{i j}(t), D_{i j}(t)$, and $r_{i j}(t)$ refer to the excitation wavelength $\lambda_{i}^{\mathrm{ex}}$ and the emission wavelength $\lambda_{j}^{\mathrm{em}}$, respectively.

The "sum" function $S_{i j}(t)$ corresponds to the total time-resolved emission of the photophysical system, is independent of the rotational diffusion and does not contain any information about the orientation of the absorption and emission transition moments. $S_{i j}(t)$ is expressed as

$$
S_{i j}(t)=\frac{1}{3} c_{j} b_{i} \exp \left(-k_{01} t\right), \quad t \geq 0
$$

where $b_{i}$ stands for the zero-time concentration of $\mathbf{1}^{*}$, which depends on the excitation wavelength $\lambda_{i}^{\mathrm{ex}}$.

The coefficient $c_{j}$ is defined as ${ }^{[21]]}$

$$
c_{j}=k_{\mathrm{F}} \int_{\Delta \lambda_{j}^{\mathrm{em}}} \rho\left(\lambda_{j}^{\mathrm{em}}\right) d \lambda^{\mathrm{em}}
$$

where $k_{\mathrm{F}}$ denotes the fluorescence rate constant of species $\mathbf{1}^{*}$. The subscript $j$ refers to the observation wavelength range $\Delta \lambda_{j}^{\mathrm{em}}$, and $\rho\left(\lambda_{j}^{\mathrm{em}}\right)$ is the spectral emission density of species $\mathbf{1}^{*}$.

The "difference" function $D_{i j}(t)$ contains information about rotational diffusion and is given by 


$$
D_{i j}(t)=\frac{8 \pi}{75} c_{j} b_{i} \exp \left(-k_{01} t\right) \boldsymbol{Y}_{2}^{M^{*}}(\overrightarrow{\mathbf{e}}) \exp \left(\mathbf{A}_{D} t\right) \boldsymbol{Y}_{2}^{M}(\overrightarrow{\mathbf{a}}), \quad t \geq 0
$$

The column vector $\boldsymbol{Y}_{2}^{M}(\overrightarrow{\mathbf{a}})$ is given by

$$
\boldsymbol{Y}_{2}^{M}(\overrightarrow{\mathbf{a}})=\left(Y_{2}^{-2}\left(\theta_{\mathrm{a}}, \phi_{\mathrm{a}}\right) \quad Y_{2}^{-1}\left(\theta_{\mathrm{a}}, \phi_{\mathrm{a}}\right) \quad Y_{2}^{0}\left(\theta_{\mathrm{a}}, \phi_{\mathrm{a}}\right) \quad Y_{2}^{1}\left(\theta_{\mathrm{a}}, \phi_{\mathrm{a}}\right) \quad Y_{2}^{2}\left(\theta_{\mathrm{a}}, \phi_{\mathrm{a}}\right)\right)^{\mathrm{T}}
$$

where $Y_{2}^{M}\left(\theta_{\mathrm{a}}, \phi_{\mathrm{a}}\right)$ are spherical harmonics, $M=-2,-1,0,1,2$, with arguments $\theta_{\mathrm{a}}$ and $\phi_{\mathrm{a}}$ which are, respectively, the polar and azimuthal angle of the absorption transition moment $\overrightarrow{\mathbf{a}}$ with respect to the molecular $(x, y, z)$ coordinate system (Figure 2). Expressions for the spherical harmonics $Y_{2}^{M}\left(\theta_{\mathrm{a}}, \phi_{\mathrm{a}}\right)$ can be found in reference [22].

\section{$<$ Figure 2>}

The $5 \times 5$ diagonal matrix $\mathbf{A}_{D}$ is given by

$$
\mathbf{A}_{D}=\operatorname{diag}\left(-\left(2 D_{\perp}+4 D_{\|}\right),-\left(5 D_{\perp}+D_{\|}\right),-6 D_{\perp},-\left(5 D_{\perp}+D_{\|}\right),-\left(2 D_{\perp}+4 D_{\|}\right)\right)
$$

$D_{\perp}$ and $D_{\|}$(see Figure 1) are the components of the rotational diffusion tensor of the cylindrically symmetric species in its molecular reference frame $(x, y, z)$, chosen such that the rotational diffusion tensor is diagonal. ${ }^{[9]}$

The row vector $\boldsymbol{Y}_{2}^{M^{*}}(\overrightarrow{\mathbf{e}})$ contains the complex conjugates of the spherical harmonics

$$
\begin{aligned}
& Y_{2}^{M^{*}}\left(\theta_{\mathrm{e}}, \phi_{\mathrm{e}}\right), M=-2,-1,0,1,2: \\
& \quad Y_{2}^{M^{*}}(\overrightarrow{\mathbf{e}})=\left(Y_{2}^{-2^{*}}\left(\theta_{\mathrm{e}}, \phi_{\mathrm{e}}\right) \quad Y_{2}^{-1^{*}}\left(\theta_{\mathrm{e}}, \phi_{\mathrm{e}}\right) \quad Y_{2}^{0}\left(\theta_{\mathrm{e}}, \phi_{\mathrm{e}}\right) \quad Y_{2}^{1^{*}}\left(\theta_{\mathrm{e}}, \phi_{\mathrm{e}}\right) \quad Y_{2}^{2^{*}}\left(\theta_{\mathrm{e}}, \phi_{\mathrm{e}}\right)\right)
\end{aligned}
$$

with arguments depending on the polar $\left(\theta_{\mathrm{e}}\right)$ and azimuthal angles $\left(\phi_{\mathrm{e}}\right)$ defined by the orientation of the emission transition moment $\overrightarrow{\mathbf{e}}$ in the molecular reference frame (Figure 2).

The time-dependent anisotropy $r_{i j}(t)[$ Eq. (2)] is given by

$$
r_{i j}(t)=\frac{8 \pi}{25} \boldsymbol{Y}_{2}^{M^{*}}(\overrightarrow{\mathbf{e}}) \exp \left(\mathbf{A}_{D} t\right) \boldsymbol{Y}_{2}^{M}(\overrightarrow{\mathbf{a}}), \quad t \geq 0
$$


Hence, we arrived at a matrix formulation of the fluorescence anisotropy $r_{i j}(t)$, ideally suited for the identifiability analysis approach using similarity transformations (see section 3). The excitation wavelength $\lambda_{i}^{\text {ex }}$ dependence of $r_{i j}(t)$ is expressed by $\boldsymbol{Y}_{2}^{M}(\overrightarrow{\mathbf{a}})$, and its emission wavelength $\lambda_{j}^{\text {em }}$ dependence by $\boldsymbol{Y}_{2}^{M}(\overrightarrow{\mathbf{e}})$. The set $\left\{\boldsymbol{Y}_{2}^{M^{*}}(\overrightarrow{\mathbf{e}}), \mathbf{A}_{D}, \boldsymbol{Y}_{2}^{M}(\overrightarrow{\mathbf{a}})\right\}$ is called a realization of $r_{i j}(t)$.

Equation (11) can be written in the common triple-exponential format:

$$
r_{i j}(t)=\beta_{1} \exp \left[-6 D_{\perp} t\right]+\beta_{2} \exp \left[-\left(5 D_{\perp}+D_{\|}\right) t\right]+\beta_{3} \exp \left[-\left(2 D_{\perp}+4 D_{\|}\right) t\right], \quad t \geq 0
$$

with

$$
\begin{aligned}
& \beta_{1}=\frac{8 \pi}{25} Y_{2}^{0}\left(\theta_{\mathrm{a}}\right) Y_{2}^{0}\left(\theta_{\mathrm{e}}\right)=0.1\left(3 \cos ^{2} \theta_{\mathrm{a}}-1\right)\left(3 \cos ^{2} \theta_{\mathrm{e}}-1\right)=0.4 P_{2}\left(\cos \theta_{\mathrm{a}}\right) P_{2}\left(\cos \theta_{\mathrm{e}}\right) \\
& \beta_{2}=\frac{8 \pi}{25}\left[Y_{2}^{-1}\left(\theta_{\mathrm{a}}, \phi_{\mathrm{a}}\right) Y_{2}^{-1^{*}}\left(\theta_{\mathrm{e}}, \phi_{\mathrm{e}}\right)+Y_{2}^{1}\left(\theta_{\mathrm{a}}\right) Y_{2}^{1^{*}}\left(\theta_{\mathrm{e}}, \phi_{\mathrm{e}}\right)\right]=0.3 \sin 2 \theta_{\mathrm{a}} \sin 2 \theta_{\mathrm{e}} \cos \varphi
\end{aligned}
$$

$$
\beta_{3}=\frac{8 \pi}{25}\left[Y_{2}^{-2}\left(\theta_{\mathrm{a}}, \phi_{\mathrm{a}}\right) Y_{2}^{-2^{*}}\left(\theta_{\mathrm{e}}, \phi_{\mathrm{e}}\right)+Y_{2}^{2}\left(\theta_{\mathrm{a}}, \phi_{\mathrm{a}}\right) Y_{2}^{2^{*}}\left(\theta_{\mathrm{e}}, \phi_{\mathrm{e}}\right)\right]=0.3 \sin ^{2} \theta_{\mathrm{a}} \sin ^{2} \theta_{\mathrm{e}} \cos 2 \varphi
$$

Equations (12)-(13) represent the classical, well-known expressions of the emission anisotropy $r_{i j}(t)$ of prolate and oblate ellipsoids, to be found in textbooks of fluorescence spectroscopy. ${ }^{[19], \text { [20] }}$

Note that the expressions for $r_{i j}(t)$ of a totally asymmetric rotor derived by Chuang and Eisenthal, ${ }^{[12]}$ Ehrenberg and Rigler, ${ }^{[13]}$, and Belford et al. ${ }^{[14]}$ reduce to Eqs. (12) and (13) in case of a cylindrically symmetric rotor.

In Eq. (13a), $P_{2}(x)=1 / 2\left(3 x^{2}-1\right)$ is the second-order Legendre polynomial. As depicted in Figure 2, $\left(\theta_{\mathrm{a}}, \phi_{\mathrm{a}}\right)$ and $\left(\theta_{\mathrm{e}}, \phi_{\mathrm{e}}\right)$ denote the orientations of respectively the absorption ( $\overrightarrow{\mathbf{a}})$ and emission $(\overrightarrow{\mathbf{e}})$ transition moments, separated by an angle $\alpha$. Note that Eqs. 
(13) are symmetrical in $\theta_{\mathrm{a}}$ and $\theta_{\mathrm{e}} . \varphi=\phi_{\mathrm{e}}-\phi_{\mathrm{a}}$ is the difference angle formed by the projections of the two transition moments in the plane perpendicular to the axis of symmetry. The angles defined by the transition moments $\overrightarrow{\mathbf{a}}$ and $\overrightarrow{\mathbf{e}}$ in the molecular reference frame satisfy the trigonometric identity ${ }^{[22]}$

$$
\cos \alpha=\cos \theta_{\mathrm{a}} \cos \theta_{\mathrm{e}}+\sin \theta_{\mathrm{a}} \sin \theta_{\mathrm{e}} \cos \varphi
$$

The addition theorem for spherical harmonics asserts that ${ }^{[22]}$

$$
P_{2}(\cos \alpha)=\frac{4 \pi}{5} \sum_{M=-2}^{2} Y_{2}^{M^{*}}\left(\theta_{\mathrm{e}}, \phi_{\mathrm{e}}\right) Y_{2}^{M}\left(\theta_{\mathrm{a}}, \phi_{\mathrm{a}}\right)=\frac{4 \pi}{5} \sum_{M=-2}^{2} Y_{2}^{M}\left(\theta_{\mathrm{e}}, \phi_{\mathrm{e}}\right) Y_{2}^{M^{*}}\left(\theta_{\mathrm{a}}, \phi_{\mathrm{a}}\right)
$$

Equation (15) in combination with Eq. (11) for $t=0$ gives

$$
r_{i j}(0)=\sum_{j=1}^{3} \beta_{j}=0.4 P_{2}(\cos \alpha)
$$

For a spherical symmetric rotor, $S_{i j}(t)$ is given by Eq. (5), just as for the cylindrically symmetric case. For a spherically symmetric rotor, where $D=D_{\perp}=D_{\|}$, the timeresolved emission anisotropy $r_{i j}(t)$ is

$$
r_{i j}(t)=0.4 P_{2}(\cos \alpha) \exp (-6 D t), \quad t \geq 0
$$

\section{Identifiability analysis}

The identification analysis is simpler if one uses $S_{i j}(t)$ [Eq. (5)] and either $D_{i j}(t)$ [Eq. (7)] or $r_{i j}(t)$ [Eq. (11)] instead of the polarized fluorescence $\delta$-response functions $I_{\| i j}(t)$ [Eq. (1a)] and $I_{\perp i j}(t)$ [Eq. (1b)]. Since the expression for the anisotropy decay $r_{i j}(t)$ is even simpler than that for $D_{i j}(t)$, we will carry out the identifiability study using the $S_{i j}(t)$ and $r_{i j}(t)$ functions.

The identification involving $S_{i j}(t)$ is trivial because $S_{i j}(t)$ is monoexponential. This leads immediately to unique values for $k_{01}$ and the product $c_{j} b_{i}$.

We will use the similarity transformation approach to the identifiability problem involving $r_{i j}(t)$ for the following two reasons. (i) Once $r_{i j}(t)$ is expressed in matrix 
form - as in Eq. (11) - similarity transformation offers a straightforward way of determining if a model is globally or locally identifiable, or not identifiable at all. A model is globally (or uniquely) identifiable if the parameters of the assumed model can be uniquely determined from the idealized experiment. If there is a finite number of alternative parameter estimates for some or all of the model parameters that fit the data, the model is locally identifiable. An infinite number of model parameter estimates fitting the data makes the considered model unidentifiable. (ii) Similarity transformation provides the explicit relationship between the true and alternative model parameters.

As mentioned before, the set $\left\{\boldsymbol{Y}_{2}^{M^{*}}(\overrightarrow{\mathbf{e}}), \mathbf{A}_{D}, \boldsymbol{Y}_{2}^{M}(\overrightarrow{\mathbf{a}})\right\}$ is called a realization of the fluorescence anisotropy $\delta$-response function $r_{i j}(t)$. The deterministic identifiability study investigates whether it is possible to find different (alternative) realizations of $r_{i j}(t)$, say $\left\{\boldsymbol{Y}_{2}^{M^{*}}\left(\overrightarrow{\mathbf{e}}^{+}\right), \mathbf{A}_{D}^{+}, \boldsymbol{Y}_{2}^{M}\left(\overrightarrow{\mathbf{a}}^{+}\right)\right\}$, so that for all times $t$

$$
r_{i j}\left(t, \boldsymbol{Y}_{2}^{M^{*}}\left(\overrightarrow{\mathbf{e}}^{+}\right), \mathbf{A}_{D}^{+}, \boldsymbol{Y}_{2}^{M}\left(\overrightarrow{\mathbf{a}}^{+}\right)\right)=r_{i j}\left(t, \boldsymbol{Y}_{2}^{M^{*}}(\overrightarrow{\mathbf{e}}), \mathbf{A}_{D}, \boldsymbol{Y}_{2}^{M}(\overrightarrow{\mathbf{a}})\right)
$$

Global (unique) identifiability is achieved when $k_{01}^{+}=k_{01}, D_{\perp}^{+}=D_{\perp}, D_{\|}^{+}=D_{\|}$, $\theta_{\mathrm{a}}^{+}=\theta_{\mathrm{a}}, \theta_{\mathrm{e}}^{+}=\theta_{\mathrm{e}}$, and $\varphi^{+}=\varphi$. The model is locally identifiable when there is a limited set of alternative $k_{01}^{+}, D_{\perp}^{+}, D_{\|}^{+}, \theta_{\mathrm{a}}^{+}, \theta_{\mathrm{e}}^{+}$, and $\varphi^{+}$. An unidentifiable model is found when there exists an infinite number of alternative $k_{01}^{+}, D_{\perp}^{+}, D_{\|}^{+}, \theta_{\mathrm{a}}^{+}, \theta_{\mathrm{e}}^{+}$, and $\varphi^{+}$

Any other (alternative) realization $\left\{\boldsymbol{Y}_{2}^{M^{*}}\left(\overrightarrow{\mathbf{e}}^{+}\right), \mathbf{A}_{D}^{+}, \boldsymbol{Y}_{2}^{M}\left(\overrightarrow{\mathbf{a}}^{+}\right)\right\}$of $r_{i j}(t)$ is related to the true set $\left\{\boldsymbol{Y}_{2}^{M^{*}}(\overrightarrow{\mathbf{e}}), \mathbf{A}_{D}, \boldsymbol{Y}_{2}^{M}(\overrightarrow{\mathbf{a}})\right\}$ via similarity transformations. ${ }^{[2], ~[3], ~[8], ~[23], ~[24], ~[25] ~}$ Two square matrices $\mathbf{A}_{D}^{+}$and $\mathbf{A}_{D}$ of the same size are said to be similar if there exists

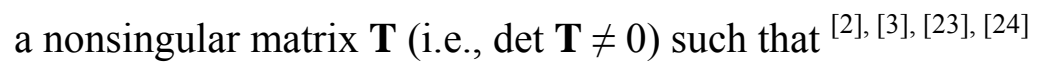




$$
\mathbf{A}_{D}^{+}=\mathbf{T}^{-1} \mathbf{A}_{D} \mathbf{T}
$$

where $\mathbf{T}^{-1}$ stands for the inverse of $\mathbf{T}$. The alternative $\boldsymbol{Y}_{2}^{M^{*}}\left(\overrightarrow{\mathbf{e}}^{+}\right)$and $\boldsymbol{Y}_{2}^{M}\left(\overrightarrow{\mathbf{a}}^{+}\right)$are given by the similarity transformations ${ }^{[2],[3]}$

$$
\begin{aligned}
& \boldsymbol{Y}_{2}^{M^{*}}\left(\overrightarrow{\mathbf{e}}^{+}\right)=\boldsymbol{Y}_{2}^{M^{*}}(\overrightarrow{\mathbf{e}}) \mathbf{T} \\
& \boldsymbol{Y}_{2}^{M}\left(\overrightarrow{\mathbf{a}}^{+}\right)=\mathbf{T}^{-1} \boldsymbol{Y}_{2}^{M}(\overrightarrow{\mathbf{a}})
\end{aligned}
$$

Equations (19)-(21) should be satisfied for each experimental condition. ${ }^{[8],[24]}$ For the models considered, the possible experimental variables are excitation wavelength $\lambda_{i}^{\text {ex }}$ and emission wavelength $\lambda_{j}^{\mathrm{em}}$. This implies that the matrix $\mathbf{T}$ should be independent of $\lambda_{i}^{\mathrm{ex}}$ and $\lambda_{j}^{\mathrm{em}}$.

Since the matrix $\mathbf{A}_{D}^{+}$[Eq. (22)] with the alternative rotational diffusion coefficients $D_{\perp}^{+}$and $D_{\|}^{+}$is a diagonal matrix similar to the square matrix $\mathbf{A}_{D}$ [Eq. (19)], the diagonal elements of $\mathbf{A}_{D}^{+}$are the corresponding eigenvalues (characteristic values) of $\mathbf{A}_{D}{ }^{[23]}$

$$
\mathbf{A}_{D}^{+}=\operatorname{diag}\left(-\left(2 D_{\perp}^{+}+4 D_{\|}^{+}\right),-\left(5 D_{\perp}^{+}+D_{\|}^{+}\right),-6 D_{\perp}^{+},-\left(5 D_{\perp}^{+}+D_{\|}^{+}\right),-\left(2 D_{\perp}^{+}+4 D_{\|}^{+}\right)\right)
$$

In other words, if $\mathbf{A}_{D}$ itself is a diagonal matrix, then $\mathbf{A}_{D}$ is similar to $\mathbf{A}_{D}^{+}$if and only if

$$
\mathbf{A}_{D}^{+}=\mathbf{A}_{D}
$$

Equation (23) is valid if the corresponding elements of $\mathbf{A}_{D}^{+}$and $\mathbf{A}_{D}$ are equal, ${ }^{[23]}$ yielding

$$
\begin{aligned}
& D_{\perp}^{+}=D_{\perp} \\
& D_{\|}^{+}=D_{\|}
\end{aligned}
$$


Since $\mathbf{A}_{D}$ has only three distinct eigenvalues, the transformation matrix $\mathbf{T}$ may not be diagonal, but is given by Eq. (25):

$$
\mathbf{T}=\left[\begin{array}{ccccc}
t_{11} & 0 & 0 & 0 & t_{15} \\
0 & t_{22} & 0 & t_{24} & 0 \\
0 & 0 & t_{33} & 0 & 0 \\
0 & t_{42} & 0 & t_{44} & 0 \\
t_{51} & 0 & 0 & 0 & t_{55}
\end{array}\right]
$$

The justification for this specific structure of the matrix $\mathbf{T}$ is based on the following. Taking into account the diagonal structure of the matrices $\mathbf{A}_{D} \equiv\left[\mathrm{a}_{i j}\right]$ and $\mathbf{A}_{D}^{+} \equiv\left[\mathrm{a}_{i j}^{+}\right]$ [Eqs. (9) and (22)], Eq. (19) can be written as

$$
t_{i j} \mathrm{a}_{j j}^{+}=\mathrm{a}_{i i} t_{i j}(i, j=1, \ldots, 5)
$$

Since $\mathrm{a}_{11}=\mathrm{a}_{55}, \mathrm{a}_{22}=\mathrm{a}_{44}, \mathrm{a}_{11} \neq \mathrm{a}_{22}, \mathrm{a}_{11} \neq \mathrm{a}_{33}$ and $\mathrm{a}_{22} \neq \mathrm{a}_{33}$ [Eq. (9)], and $\mathbf{A}_{D}^{+}=\mathbf{A}_{D}$ [Eq. (23)], Eq. (26) yields for $j=1$

$$
\begin{aligned}
& t_{21}=0 \\
& t_{31}=0 \\
& t_{41}=0
\end{aligned}
$$

Repeating this reasoning for the remaining values of $j(2, \ldots, 5)$, one can deduce that the form of the transformation matrix $\mathbf{T}$ is given by Eq. (25).

The matrix multiplications of Eq. (20) (for emission) with $\mathbf{T}$ given by Eq. (25) yield

$$
\begin{aligned}
& Y_{2}^{-2^{*}}\left(\theta_{\mathrm{e}}^{+}, \phi_{\mathrm{e}}^{+}\right)=t_{11} Y_{2}^{-2^{*}}\left(\theta_{\mathrm{e}}, \phi_{\mathrm{e}}\right)+t_{51} Y_{2}^{2^{*}}\left(\theta_{\mathrm{e}}, \phi_{\mathrm{e}}\right) \\
& Y_{2}^{-1^{*}}\left(\theta_{\mathrm{e}}^{+}, \phi_{\mathrm{e}}^{+}\right)=t_{22} Y_{2}^{-1^{*}}\left(\theta_{\mathrm{e}}, \phi_{\mathrm{e}}\right)+t_{42} Y_{2}^{1^{*}}\left(\theta_{\mathrm{e}}, \phi_{\mathrm{e}}\right) \\
& Y_{2}^{0}\left(\theta_{\mathrm{e}}^{+}, \phi_{\mathrm{e}}^{+}\right)=t_{33} Y_{2}^{0}\left(\theta_{\mathrm{e}}, \phi_{\mathrm{e}}\right) \\
& Y_{2}^{1^{*}}\left(\theta_{\mathrm{e}}^{+}, \phi_{\mathrm{e}}^{+}\right)=t_{24} Y_{2}^{-1^{*}}\left(\theta_{\mathrm{e}}, \phi_{\mathrm{e}}\right)+t_{44} Y_{2}^{1^{*}}\left(\theta_{\mathrm{e}}, \phi_{\mathrm{e}}\right)
\end{aligned}
$$




$$
Y_{2}^{2^{*}}\left(\theta_{\mathrm{e}}^{+}, \phi_{\mathrm{e}}^{+}\right)=t_{15} Y_{2}^{-2^{*}}\left(\theta_{\mathrm{e}}, \phi_{\mathrm{e}}\right)+t_{55} Y_{2}^{2^{*}}\left(\theta_{\mathrm{e}}, \phi_{\mathrm{e}}\right)
$$

Similarly, the matrix multiplications of Eq. (21) (for absorption) with $\mathbf{T}$ given by Eq. (25) yield

$$
\begin{aligned}
& t_{11} Y_{2}^{-2}\left(\theta_{\mathrm{a}}^{+}, \phi_{\mathrm{a}}^{+}\right)+t_{15} Y_{2}^{2}\left(\theta_{\mathrm{a}}^{+}, \phi_{\mathrm{a}}^{+}\right)=Y_{2}^{-2}\left(\theta_{\mathrm{a}}, \phi_{\mathrm{a}}\right) \\
& t_{22} Y_{2}^{-1}\left(\theta_{\mathrm{a}}^{+}, \phi_{\mathrm{a}}^{+}\right)+t_{24} Y_{2}^{1}\left(\theta_{\mathrm{a}}^{+}, \phi_{\mathrm{a}}^{+}\right)=Y_{2}^{-1}\left(\theta_{\mathrm{a}}, \phi_{\mathrm{a}}\right) \\
& t_{33} Y_{2}^{0}\left(\theta_{\mathrm{a}}^{+}, \phi_{\mathrm{a}}^{+}\right)=Y_{2}^{0}\left(\theta_{\mathrm{a}}, \phi_{\mathrm{a}}\right) \\
& t_{42} Y_{2}^{-1}\left(\theta_{\mathrm{a}}^{+}, \phi_{\mathrm{a}}^{+}\right)+t_{44} Y_{2}^{1}\left(\theta_{\mathrm{a}}^{+}, \phi_{\mathrm{a}}^{+}\right)=Y_{2}^{1}\left(\theta_{\mathrm{a}}, \phi_{\mathrm{a}}\right) \\
& t_{51} Y_{2}^{-2}\left(\theta_{\mathrm{a}}^{+}, \phi_{\mathrm{a}}^{+}\right)+t_{55} Y_{2}^{2}\left(\theta_{\mathrm{a}}^{+}, \phi_{\mathrm{a}}^{+}\right)=Y_{2}^{2}\left(\theta_{\mathrm{a}}, \phi_{\mathrm{a}}\right)
\end{aligned}
$$

Multiplication of the respective lhs and rhs of Eqs. (28c) and (29c) eliminates $t_{33}$ and yields

$$
Y_{2}^{0}\left(\theta_{\mathrm{a}}^{+}, \phi_{\mathrm{a}}^{+}\right) Y_{2}^{0}\left(\theta_{\mathrm{e}}^{+}, \phi_{\mathrm{e}}^{+}\right)=Y_{2}^{0}\left(\theta_{\mathrm{a}}, \phi_{\mathrm{a}}\right) Y_{2}^{0}\left(\theta_{\mathrm{e}}, \phi_{\mathrm{e}}\right)
$$

Equation (30a) corresponds to the identifiability equation derived from Eq. (13a):

$$
\left(3 \cos ^{2} \theta_{\mathrm{a}}^{+}-1\right)\left(3 \cos ^{2} \theta_{\mathrm{e}}^{+}-1\right)=\left(3 \cos ^{2} \theta_{\mathrm{a}}-1\right)\left(3 \cos ^{2} \theta_{\mathrm{e}}-1\right)
$$

From Eq. (28b), we have

$$
t_{42}=\frac{Y_{2}^{-1^{*}}\left(\theta_{\mathrm{e}}^{+}, \phi_{\mathrm{e}}^{+}\right)-t_{22} Y_{2}^{-*^{*}}\left(\theta_{\mathrm{e}}, \phi_{\mathrm{e}}\right)}{Y_{2}^{1^{*}}\left(\theta_{\mathrm{e}}, \phi_{\mathrm{e}}\right)}
$$

and substitution of $t_{42}$ in Eq. (29d) gives

$$
-t_{22} Y_{2}^{-1}\left(\theta_{\mathrm{a}}^{+}, \phi_{\mathrm{a}}^{+}\right) Y_{2}^{-1^{*}}\left(\theta_{\mathrm{e}}, \phi_{\mathrm{e}}\right)+t_{44} Y_{2}^{1}\left(\theta_{\mathrm{a}}^{+}, \phi_{\mathrm{a}}^{+}\right) Y_{2}^{1^{*}}\left(\theta_{\mathrm{e}}, \phi_{\mathrm{e}}\right)=Y_{2}^{1}\left(\theta_{\mathrm{a}}, \phi_{\mathrm{a}}\right) Y_{2}^{1^{*}}\left(\theta_{\mathrm{e}}, \phi_{\mathrm{e}}\right)-Y_{2}^{-1}\left(\theta_{\mathrm{a}}^{+}, \phi_{\mathrm{a}}^{+}\right) Y_{2}^{-1^{*}}\left(\theta_{\mathrm{e}}^{+}, \phi_{\mathrm{e}}^{+}\right)
$$

Analogously, from Eq. (28d), we have

$$
t_{24}=\frac{Y_{2}^{1^{*}}\left(\theta_{\mathrm{e}}^{+}, \phi_{\mathrm{e}}^{+}\right)-t_{44} Y_{2}^{1^{*}}\left(\theta_{\mathrm{e}}, \phi_{\mathrm{e}}\right)}{Y_{2}^{-1^{*}}\left(\theta_{\mathrm{e}}, \phi_{\mathrm{e}}\right)}
$$

and substitution of $t_{24}$ in Eq. (29b) gives $t_{22} Y_{2}^{-1}\left(\theta_{\mathrm{a}}^{+}, \phi_{\mathrm{a}}^{+}\right) Y_{2}^{-1^{*}}\left(\theta_{\mathrm{e}}, \phi_{\mathrm{e}}\right)-t_{44} Y_{2}^{1}\left(\theta_{\mathrm{a}}^{+}, \phi_{\mathrm{a}}^{+}\right) Y_{2}^{*^{*}}\left(\theta_{\mathrm{e}}, \phi_{\mathrm{e}}\right)=Y_{2}^{-1}\left(\theta_{\mathrm{a}}, \phi_{\mathrm{a}}\right) Y_{2}^{-1^{*}}\left(\theta_{\mathrm{e}}, \phi_{\mathrm{e}}\right)-Y_{2}^{1}\left(\theta_{\mathrm{a}}^{+}, \phi_{\mathrm{a}}^{+}\right) Y_{2}^{1^{*}}\left(\theta_{\mathrm{e}}^{+}, \phi_{\mathrm{e}}^{+}\right)$ 
From Eqs. (31b) and (32b), we have

$$
Y_{2}^{-1}\left(\theta_{\mathrm{a}}^{+}, \phi_{\mathrm{a}}^{+}\right) Y_{2}^{-1^{*}}\left(\theta_{\mathrm{e}}^{+}, \phi_{\mathrm{e}}^{+}\right)+Y_{2}^{1}\left(\theta_{\mathrm{a}}^{+}, \phi_{\mathrm{a}}^{+}\right) Y_{2}^{1^{*}}\left(\theta_{\mathrm{e}}^{+}, \phi_{\mathrm{e}}^{+}\right)=Y_{2}^{-1}\left(\theta_{\mathrm{a}}, \phi_{\mathrm{a}}\right) Y_{2}^{-1^{*}}\left(\theta_{\mathrm{e}}, \phi_{\mathrm{e}}\right)+Y_{2}^{1}\left(\theta_{\mathrm{a}}, \phi_{\mathrm{a}}\right) Y_{2}^{1^{*}}\left(\theta_{\mathrm{e}}, \phi_{\mathrm{e}}\right)
$$

which is the same identifiability equation as from Eq. (13b):

$$
\sin 2 \theta_{\mathrm{a}}^{+} \sin 2 \theta_{\mathrm{e}}^{+} \cos \varphi^{+}=\sin 2 \theta_{\mathrm{a}} \sin 2 \theta_{\mathrm{e}} \cos \varphi
$$

Similarly, from Eqs. (28a), (28e), (29a), and (29e), we get, after some rearrangement

$$
Y_{2}^{-2}\left(\theta_{\mathrm{a}}^{+}, \phi_{\mathrm{a}}^{+}\right) Y_{2}^{-2^{*}}\left(\theta_{\mathrm{e}}^{+}, \phi_{\mathrm{e}}^{+}\right)+Y_{2}^{2}\left(\theta_{\mathrm{a}}^{+}, \phi_{\mathrm{a}}^{+}\right) Y_{2}^{2^{*}}\left(\theta_{\mathrm{e}}^{+}, \phi_{\mathrm{e}}^{+}\right)=Y_{2}^{-2}\left(\theta_{\mathrm{a}}, \phi_{\mathrm{a}}\right) Y_{2}^{-2^{*}}\left(\theta_{\mathrm{e}}, \phi_{\mathrm{e}}\right)+Y_{2}^{2}\left(\theta_{\mathrm{a}}, \phi_{\mathrm{a}}\right) Y_{2}^{2^{*}}\left(\theta_{\mathrm{e}}, \phi_{\mathrm{e}}\right)
$$

which is the same identifiability equation as from Eq. (13c):

$$
\sin ^{2} \theta_{\mathrm{a}}^{+} \sin ^{2} \theta_{\mathrm{e}}^{+} \cos 2 \varphi^{+}=\sin ^{2} \theta_{\mathrm{a}} \sin ^{2} \theta_{\mathrm{e}} \cos 2 \varphi
$$

Using the symbolic mathematics program MAPLE V (Waterloo Maple Inc.), it can be shown that, for the polar angles in the interval $[0, \pi / 2]$ and for the azimuthal angles in the interval $[0, \pi]$, there are four sets of solutions for Eqs. (30b), (33b), and (34b) in terms of the three unknowns $\theta_{\mathrm{a}}^{+}, \theta_{\mathrm{e}}^{+}$, and $\varphi^{+}$:

(i) $\theta_{\mathrm{a}}^{+}=\theta_{\mathrm{a}}, \theta_{\mathrm{e}}^{+}=\theta_{\mathrm{e}}, \varphi^{+}=\varphi$

(ii) $\theta_{\mathrm{a}}^{+}=\theta_{\mathrm{e}}, \theta_{\mathrm{e}}^{+}=\theta_{\mathrm{a}}, \varphi^{+}=\varphi$

(iii) $\theta_{\mathrm{a}}^{+}=\arcsin \sqrt{1-\frac{1}{2} f\left(\theta_{\mathrm{a}}, \theta_{\mathrm{e}}, \varphi\right)+\frac{1}{2}\left\{f^{2}\left(\theta_{\mathrm{a}}, \theta_{\mathrm{e}}, \varphi\right)-4 \sin ^{2} \theta_{\mathrm{a}} \sin ^{2} \theta_{\mathrm{e}} \cos ^{2} \varphi\right\}^{1 / 2}}$

$$
\begin{aligned}
& \theta_{\mathrm{e}}^{+}=\arcsin \sqrt{1-\frac{1}{2} f\left(\theta_{\mathrm{a}}, \theta_{\mathrm{e}}, \varphi\right)-\frac{1}{2}\left\{f^{2}\left(\theta_{\mathrm{a}}, \theta_{\mathrm{e}}, \varphi\right)-4 \sin ^{2} \theta_{\mathrm{a}} \sin ^{2} \theta_{\mathrm{e}} \cos ^{2} \varphi\right\}^{1 / 2}} \\
& \varphi^{+}=\arccos \sqrt{\frac{\left(\sin ^{2} \theta_{\mathrm{a}}-1\right)\left(\sin ^{2} \theta_{\mathrm{e}}-1\right)}{1+\sin ^{2} \theta_{\mathrm{a}} \sin ^{2} \theta_{\mathrm{e}} \cos ^{2} \varphi-f\left(\theta_{\mathrm{a}}, \theta_{\mathrm{e}}, \varphi\right)}}
\end{aligned}
$$

where

$$
f\left(\theta_{\mathrm{a}}, \theta_{\mathrm{e}}, \varphi\right)=2 \sin ^{2} \theta_{\mathrm{a}}+2 \sin ^{2} \theta_{\mathrm{e}}-3 \sin ^{2} \theta_{\mathrm{a}} \sin ^{2} \theta_{\mathrm{e}} \sin ^{2} \varphi-1
$$




$$
\text { iv) } \theta_{\mathrm{a}}^{+}=\arcsin \sqrt{1-\frac{1}{2} f\left(\theta_{\mathrm{a}}, \theta_{\mathrm{e}}, \varphi\right)-\frac{1}{2}\left\{f^{2}\left(\theta_{\mathrm{a}}, \theta_{\mathrm{e}}, \varphi\right)-4 \sin ^{2} \theta_{\mathrm{a}} \sin ^{2} \theta_{\mathrm{e}} \cos ^{2} \varphi\right\}^{1 / 2}}
$$

$$
\theta_{\mathrm{e}}^{+}=\arcsin \sqrt{1-\frac{1}{2} f\left(\theta_{\mathrm{a}}, \theta_{\mathrm{e}}, \varphi\right)+\frac{1}{2}\left\{f^{2}\left(\theta_{\mathrm{a}}, \theta_{\mathrm{e}}, \varphi\right)-4 \sin ^{2} \theta_{\mathrm{a}} \sin ^{2} \theta_{\mathrm{e}} \cos ^{2} \varphi\right\}^{1 / 2}}
$$

with $\varphi^{+}$given by Eq. (37c) and $f\left(\theta_{\mathrm{a}}, \theta_{\mathrm{e}}, \varphi\right)$ given by Eq. (38).

Since there are several sets of alternative $\left\{\theta_{\mathrm{a}}^{+}, \theta_{\mathrm{e}}^{+}, \varphi^{+}\right\}$, the model is locally identifiable in terms of the angles $\theta_{\mathrm{a}}, \theta_{\mathrm{e}}$, and $\varphi$. Sets (i) and (ii) [Eqs. (35) and (36)] express the alternative angles $\theta^{+}$as a function of $\theta$ only, and $\varphi^{+}$as a function of $\varphi$ only. The alternative polar angles are interrelated by swapping the labels of the absorption and emission transition moments, indicating that no distinction can be made between the orientations of the absorption and emission transition moments. This is illustrated in Figure 3, where two cones (two angles $\theta$ ) can be determined: one for the absorption transition moment and one for the emission transition moment. Hence, one cannot assign a particular polar angle to a specific transition moment. In sets (iii) and (iv) [Eqs. (37)-(39)], the alternative polar and azimuthal angles are function of all true angles. The alternative angles $\theta^{+}$in sets (iii) and (iv) are obtained by swapping the labels of the absorption and emission transition moments, defining an additional set of two cone surfaces. It must emphasized that acceptable alternatives $\left\{\theta_{\mathrm{a}}^{+}, \theta_{\mathrm{e}}^{+}, \varphi^{+}\right\}$, calculated with Eqs. (37)-(39), can only be obtained for confined subspaces $\left\{\theta_{\mathrm{a}}, \theta_{\mathrm{e}}, \varphi\right\}$. The boundaries of these coupled regions can be obtained numerically. The expressions for sets (i)-(iv) are for the polar angles restricted to [0, $\pi / 2]$ and for the difference of the azimuthal angles to $[0, \pi]$. Since there is no head or tail with respect to the transition moment, the polar angle $\theta,-\theta, \pi-\theta$, and $\pi+\theta$ all refer to the same orientation. For example, for set (i) some alternatives are given by 
$\left\{\theta_{\mathrm{a}}^{+}=\theta_{\mathrm{a}}, \quad \theta_{\mathrm{e}}^{+}=-\theta_{\mathrm{e}}, \quad \varphi^{+}=\pi-\varphi\right\}, \quad\left\{\theta_{\mathrm{a}}^{+}=-\theta_{\mathrm{a}}, \quad \theta_{\mathrm{e}}^{+}=\theta_{\mathrm{e}}, \quad \varphi^{+}=\pi-\varphi\right\}, \quad\left\{\theta_{\mathrm{a}}^{+}=-\theta_{\mathrm{a}}\right.$,

$\left.\theta_{\mathrm{e}}^{+}=-\theta_{\mathrm{e}}, \varphi^{+}=\varphi\right\}, \ldots$ The value of the "difference" angle $\varphi$ between the projections of the transition moments in the $(x, y)$ plane is such that $\cos \alpha$ is uniquely determined.

\section{$<$ Figure $3>$}

To summarize, the identifiability analysis involving both $S_{i j}(t)$ and $r_{i j}(t)$ shows that the model for one excited-state processes with coupled rotational diffusion for a cylindrically symmetric ellipsoid is uniquely identifiable in terms of the deactivation rate constant $k_{01}$, the rotational diffusion coefficients $D_{\perp}$ and $D_{\|}$, and the absolute value of the polar angle $\alpha$ between the absorption $(\overrightarrow{\mathbf{a}})$ and emission $(\overrightarrow{\mathbf{e}})$ transition moments. The angle $\alpha$ between $\overrightarrow{\mathbf{a}}$ and $\overrightarrow{\mathbf{e}}$ is related to the zero-time anisotropy $r_{i j}(0)$ [Eq. (16)], and its absolute value is given by

$$
|\alpha|=\arccos \sqrt{\frac{5}{3} r_{i j}(0)+\frac{1}{3}}
$$

The polar angles $\theta_{\mathrm{a}}$ and $\theta_{\mathrm{e}}$, and the difference azimuthal angle $\varphi$ formed by the projections of the two transition moments in the plane perpendicular to the symmetry axis are locally identifiable.

Now we will consider some limiting cases. When the absorption and emission transition moments are collinear $\left(\theta_{\mathrm{a}}=\theta_{\mathrm{e}}=\theta\right.$ and $\left.\varphi=0\right)$, the pre-exponential factors $\beta_{i}$ $\left[i=1-3\right.$, Eq. (13)] in the expression of $r_{i j}(t)$ [Eq. (12)] simplify

$$
\begin{aligned}
& \beta_{1}=0.1\left(3 \cos ^{2} \theta-1\right)^{2} \\
& \beta_{2}=0.3 \sin ^{2} 2 \theta \\
& \beta_{3}=0.3 \sin ^{4} \theta
\end{aligned}
$$


Using elementary properties of the trigonometric functions the identifiability equations corresponding to Eqs. (41a-c) can be rewritten, respectively, in the form of Eqs. $(42 \mathrm{a}-\mathrm{c})$ :

$$
\begin{aligned}
& 9 \cos ^{4} \theta^{+}-6 \cos ^{2} \theta^{+}=9 \cos ^{4} \theta-6 \cos ^{2} \theta \\
& -\cos ^{4} \theta^{+}+\cos ^{2} \theta^{+}=-\cos ^{4} \theta+\cos ^{2} \theta \\
& \cos ^{4} \theta^{+}-2 \cos ^{2} \theta^{+}=\cos ^{4} \theta-2 \cos ^{2} \theta
\end{aligned}
$$

The requirement that Eqs. (42) should form a consistent set of equations indicates that the polar angle $\theta$ is uniquely recovered within the interval $[0, \pi / 2]$.

For a cylindrically symmetric rotor for which the absorption or emission transition moment is parallel to the symmetry axis $\left(\theta_{\mathrm{a}}=0\right.$ or $\left.\theta_{\mathrm{e}}=\theta\right)$, the anisotropy decay $r_{i j}(t)$ is mono-exponential

$$
r_{i j}(t)=\beta_{1} \exp \left[-6 D_{\perp} t\right], \quad t \geq 0
$$

with $\beta_{1}$ given by

$$
\beta_{1}=0.2\left(3 \cos ^{2} \theta_{\mathrm{a}}-1\right) \text { if } \theta_{\mathrm{e}}=0
$$

or

$$
\beta_{1}=0.2\left(3 \cos ^{2} \theta_{\mathrm{e}}-1\right) \text { if } \theta_{\mathrm{a}}=0
$$

Since $r_{i j}(t)$ is mono-exponential, one immediately has $D_{\perp}^{+}=D_{\perp}$ and $\theta_{\mathrm{a}}^{+}=\theta$ and $\theta_{\mathrm{e}}^{+}=0$, or $\theta_{\mathrm{e}}^{+}=\theta$ and $\theta_{\mathrm{a}}^{+}=0$.

If $\beta_{1}=0.4$, both transition moments are along the symmetry axis, which implies identifiability.

When the absorption and emission transition moments lie in a plane perpendicular to the axis of symmetry $\left(\theta_{\mathrm{a}}=\theta_{\mathrm{e}}=\theta=\pi / 2\right)$, the anisotropy decay $r_{i j}(t)$ [Eq. (12)] is biexponential with $\beta_{1}=0.1, \beta_{2}=0$, and $\beta_{3}=0.3 \cos 2 \varphi$. If the angle $\varphi$ is $\pi / 4\left(45^{\circ}\right)$, the expression of $r_{i j}(t)$ reduces to Eq. (43a) with $\beta_{1}=0.1$. It is clear that in the latter cases 
the model is identifiable, apart from the swapping of the azimuthal angles for the absorption and emission transition moments

Finally, we consider the case of a spherically symmetric rotor $\left(D=D_{\perp}=D_{\|}\right)$. The expression for $S_{i j}(t)$ is identical with that for the cylindrically symmetric case [Eq. (5)], so that $k_{01}$ and the product $c_{j} b_{i}$ are uniquely identified. Since the expression of $r_{i j}(t)$ is monoexponential [Eq. (17)], one immediately has $D^{+}=D$ and $\alpha^{+}=\alpha$, demonstrating that all relevant parameters are uniquely determined.

\section{Discussion and conclusions}

We have shown that the similarity transformation approach can be applied successfully to the deterministic identifiability analysis of kinetic models of one-state excited-state events in isotropic environments coupled with rotational diffusion described by Brownian reorientation. The similarity transformation approach has the extra merit of providing the explicit relationships between the true and alternative model parameters. We have used the "sum" $S(t)=I_{\|}(t)+2 I_{\perp}(t)$ and the fluorescence anisotropy $\delta$-response function $r(t)=D(t) / S(t)$ with $D(t)=I_{\|}(t)-I_{\perp}(t)$ in the identifiability. $S(t)$ describes the time dependence of the total fluorescence and contains information only about the deactivation of the excited state as a whole (expressed by the composite rate constant $k_{01}$ ). The time-resolved fluorescence anisotropy $r(t)$ expresses the rotational diffusion in terms of rotational diffusion constants and the angles defining the orientation of the absorption and emission transition moments. As $r(t)$ can be written as a simple function of the vectors $\boldsymbol{Y}_{2}^{M^{*}}(\overrightarrow{\mathbf{e}})$ and $\boldsymbol{Y}_{2}^{M}(\overrightarrow{\mathbf{a}})$, and the matrix $\mathbf{A}_{D}$, the identifiability analysis is simpler if one uses $r(t)$ instead of $I_{\|}(t)$ and $I_{\perp}(t)$. 
The identifiability analysis of $r(t)$ for the cylindrically symmetric rotor demonstrates that the rotational diffusion coefficients $D_{\|}$and $D_{\perp}$ can be determined, in addition to the polar angle $\alpha$ between the absorption and emission transition moments. It must be stressed that the absolute orientations of the absorption and emission transition moments are not uniquely known. Indeed, the polar angles $\theta_{\mathrm{a}}$ and $\theta_{\mathrm{e}}$ cannot be unambiguously be assigned to the two transition moments. Possible $\theta_{\mathrm{a}}, \theta_{\mathrm{e}}$ ambiguity can be resolved by considering two different excitation wavelengths yielding absorption transition moments with different orientations while the emission transition moment remains the same.

For the spherically symmetric rotor, the rotational diffusion constant $D$ and the angle $\alpha$ between the absorption and emission transition moments can be determined from the monoexponential fluorescence anisotropy decay $r(t)$.

\section{Acknowledgments}

Dr. Robert E. Dale (Kings College, London, U. K.) is thanked for helpful discussions. The continuing support by the Fonds voor Wetenschappelijk Onderzoek - Vlaanderen (FWO) is gratefully acknowledged. Part of the work was supported by DWTC (Belgium) through an IAP-V-03 grant.

\section{Figure captions}

Figure 1. Graphic representation of a one-state excited-state process, including rotation. Species $\mathbf{1}^{*}$ is pictured as being initially excited from its ground state $\mathbf{1}$ by an infinitely short linearly polarized light pulse at wavelength $\lambda_{i}^{\text {ex }}$ in a unique absorption band. The excited-state deactivation is described by the rate constant $k_{01}$. Simultaneously the species rotates with rate constants determined by the rotational 
diffusion tensor. The polarized emission depends on the relative orientation of its emission transition moment (with unit vector $\overrightarrow{\mathbf{e}}$ ) at the instant of emission with respect to the absorption transition moment (with unit vector $\overrightarrow{\mathbf{a}}$ ) at the instant of excitation.

Figure 2. Definition of the polar angles $\theta_{\mathrm{a}}$ and $\theta_{\mathrm{e}}$, the azimuthal angles $\phi_{\mathrm{a}}$ and $\phi_{\mathrm{e}}$, and the difference angles $\alpha$ and $\varphi$ when the absorption $(\overrightarrow{\mathbf{a}})$ and emission $(\overrightarrow{\mathbf{e}})$ transition moments are not parallel.

Figure 3. The two double-sided cones defining the absorption and emission transition moments. It must be emphasized that the angles $\theta,-\theta, \pi-\theta$, and $\pi+\theta$ all define the same double-sided cone. 

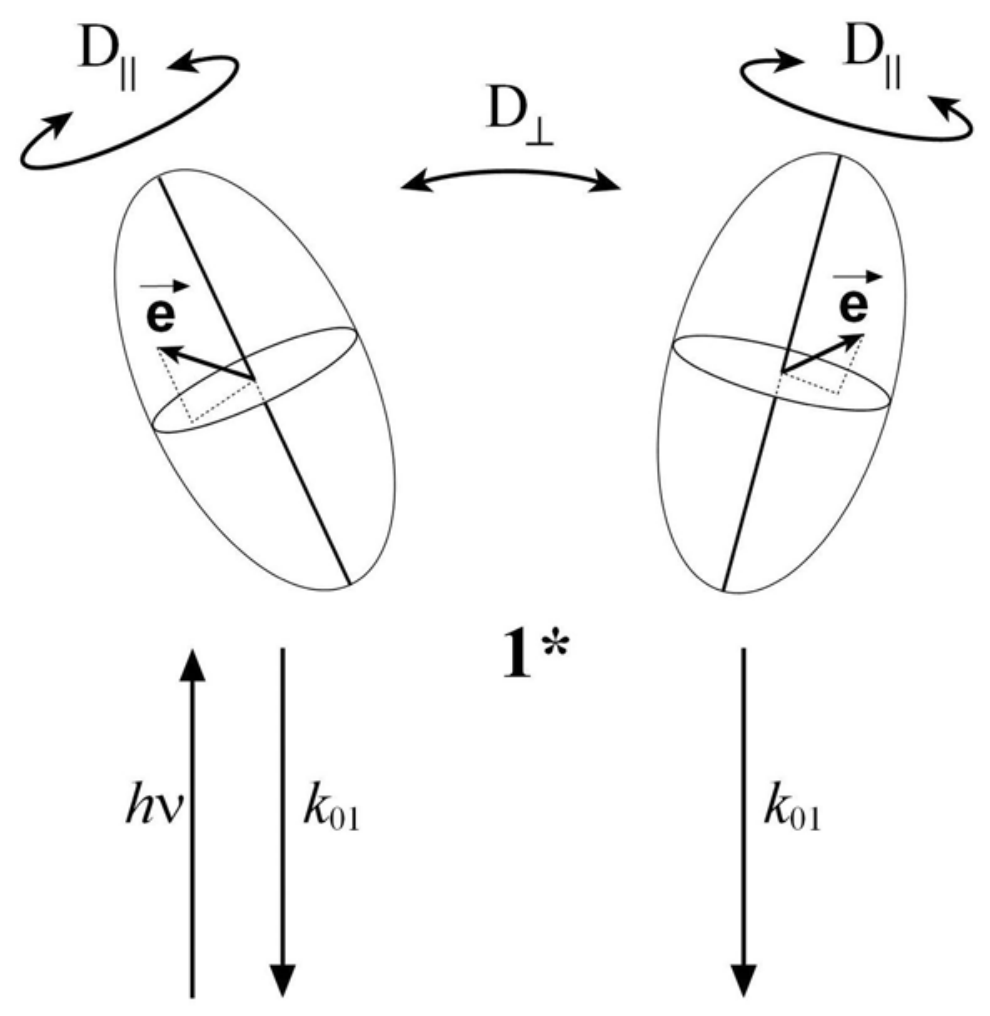

1*
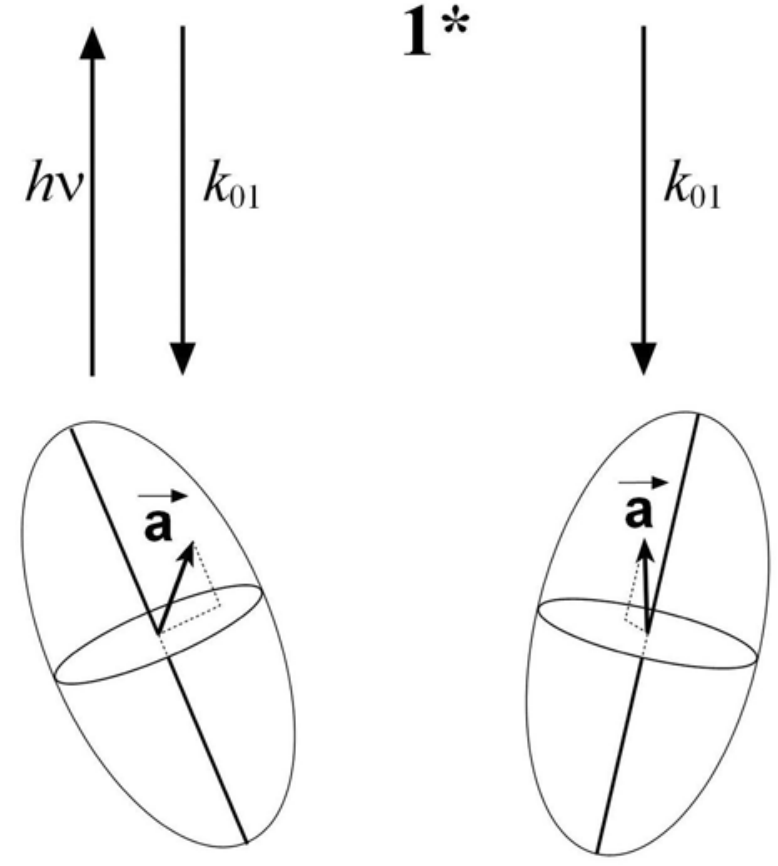

1

Figure $1 \uparrow$ 


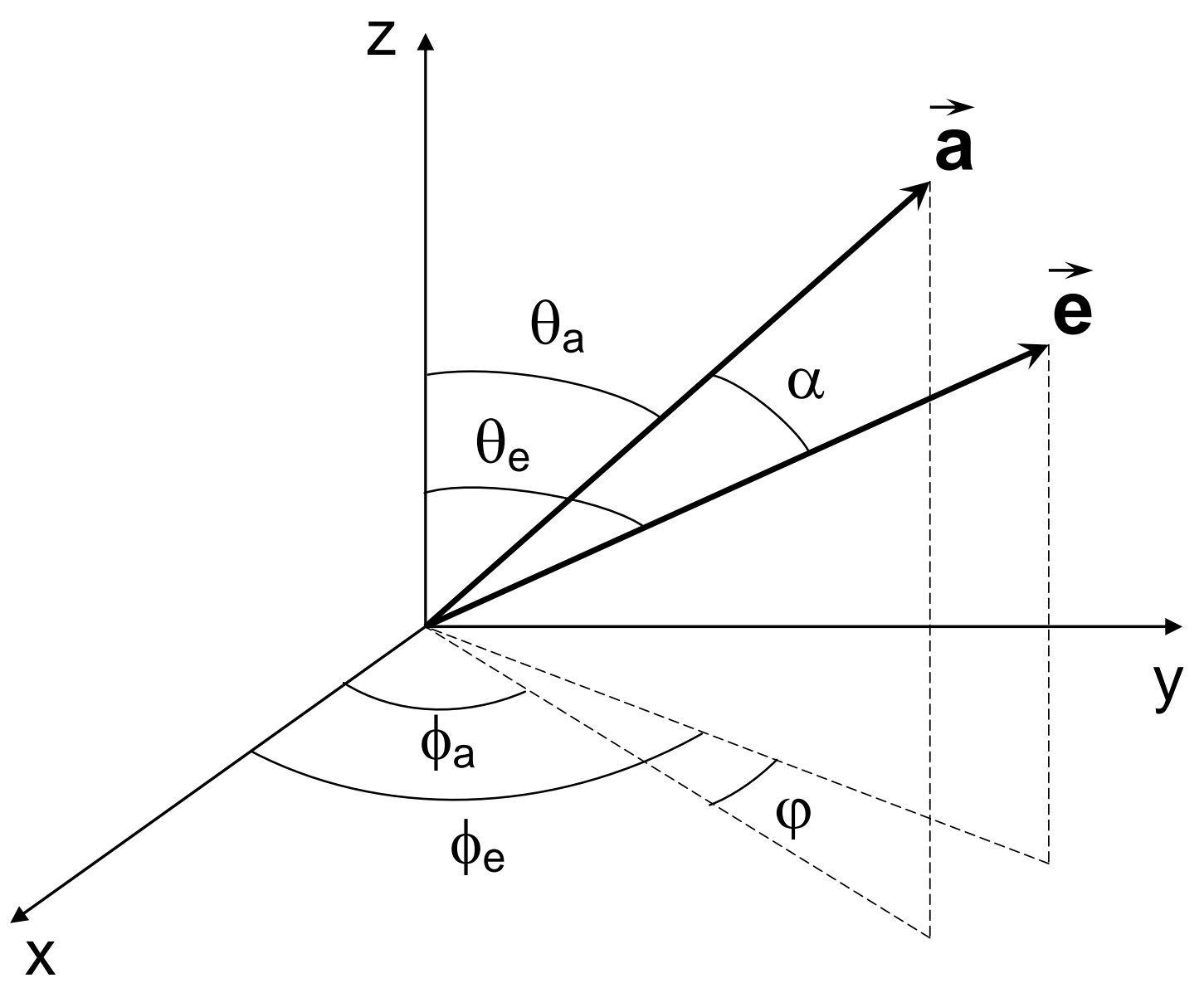

Figure $2 \uparrow$ 


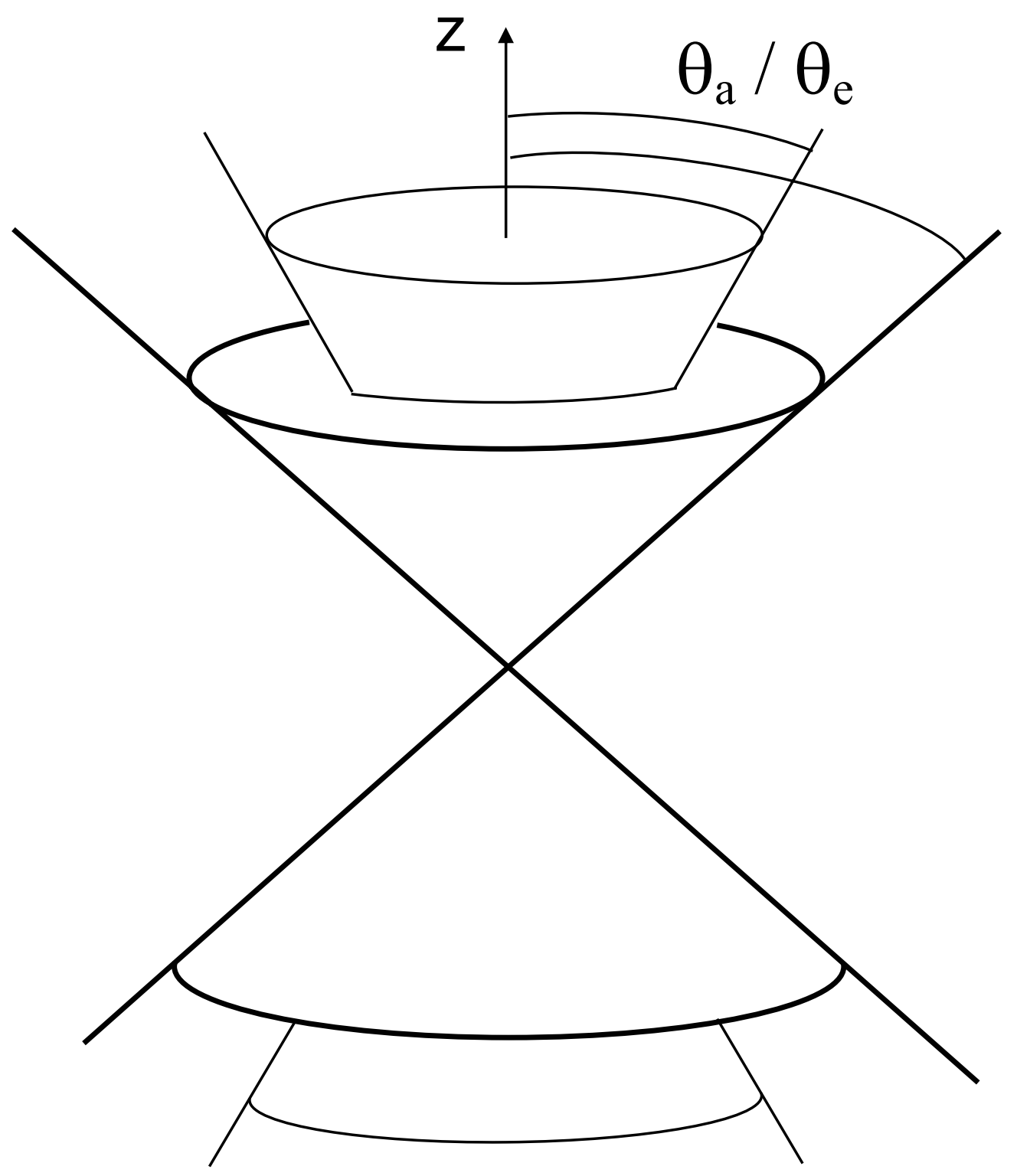

Figure $3 \uparrow$ 


\section{References}

[1] J. A. Jacquez, Compartmental Analysis in Biology and Medicine, 3rd Ed., BioMedware, Ann Arbor, 1996.

[2] K. Godfrey, Compartmental Models and Their Application, Academic Press, New York, 1983.

[3] D. H. Anderson, Compartmental Modeling and Tracer Kinetics, in: Lecture Notes in Biomathematics, Vol. 50, Springer-Verlag, Berlin, 1983.

[4] N. Boens, M. Ameloot, Internatl. J. Quantum Chem. 106 (2006) 300.

[5] J. M. Beechem, M. Ameloot, L. Brand, Chem. Phys. Lett. 120 (1985) 466.

[6] J. M. Beechem, M. Ameloot, L. Brand, Anal. Instrum. 14 (1985) 379.

[7] M. Ameloot, J. M. Beechem, L. Brand, Chem. Phys. Lett. 129 (1986) 211.

[8] N. Boens, J. Szubiakowski, E. Novikov, M. Ameloot, J. Chem. Phys. 112 (2000) 8260.

[9] J. P. Szubiakowski, R. E. Dale, N. Boens, M. Ameloot, J. Chem. Phys. 121 (2004) 7829.

[10] N. Boens, E. Novikov, M. Ameloot, J. Phys. Chem. A 109 (2005) 7024.

[11] N. Boens, E. Novikov, J. P. Szubiakowski, M. Ameloot, J. Phys. Chem. A 109 (2005) 11655.

[12] T. J. Chuang, K. B. Eisenthal, J. Chem. Phys. 57 (1972) 5094.

[13] M. Ehrenberg, R. Rigler, Chem. Phys. Lett. 14 (1972) 539.

[14] G. G. Belford, R. L. Belford, G. Weber, Proc. Natl. Acad. Sci. USA 69 (1972) 1392.

[15] L. D. Favro, Phys. Rev. 119 (1960) 53.

[16] K. Razi Naqvi, Chem. Phys. Lett. 136 (1987) 407.

[17] D. W. Piston, E. Gratton, Biophys. J. 56 (1989) 1083.

[18] G. Weber, J. Chem. Phys. 55 (1971) 2399.

[19] B. Valeur, Molecular Fluorescence. Principles and Applications, Wiley-VCH, Weinheim, 2002.

[20] J. R. Lakowicz, Principles of Fluorescence Spectroscopy, 2nd Ed., Kluwer, New York, 1999.

[21] M. Ameloot, N. Boens, R. Andriessen, V. Van den Bergh, F. C. De Schryver, J. Phys. Chem. 95 (1991) 2041-2047.

[22] G. Arfken, Mathematical Methods for Physicists, 3rd Ed., Academic Press, San Diego, 1985.

[23] A. L. Rabenstein, Elementary Differential Equations with Linear Algebra, 3rd Ed., Harcourt Brace Jovanovich, San Diego, 1982.

[24] S. Vajda, H. Rabitz, J. Phys. Chem. 92 (1988) 701. 
[25] M. J. Chappell, K. R. Godfrey, S. Vajda, Math. Biosci. 102 (1990) 41. 